\title{
A FIRST PASSAGE PROBLEM AND ITS APPLICATIONS TO THE ANALYSIS OF A CLASS OF STOCHASTIC MODELS ${ }^{1}$
}

\author{
LEV ABOLNIKOV \\ Department of Mathematics \\ Loyola Marymount University \\ Los Angeles, CA 90045, USA \\ JEWGENI H. DSHALALOW \\ Department of Applied Mathematics \\ Florida Institute of Technology \\ Melbourne, FL 32901, USA
}

\begin{abstract}
A problem of the first passage of a cumulative random process with generally distributed discrete or continuous increments over a fixed level is considered in the article as an essential part of the analysis of a class of stochastic models (bulk queueing systems, inventory control and dam models).

Using direct probability methods the authors find various characteristics of this problem: the magnitude of the first excess of the process over a fixed level, the shortage before the first excess, the levels of the first and prefirst excesses, the index of the first excess and others. The results obtained are illustrated by a number of numerical examples and then are applied to a bulk queueing system with a service delay discipline.
\end{abstract}

Key words: first passage problem, fluctuation theory, delayed renewal process, first excess level, pre-first excess level, shortage before the first excess, index of the first excess, bulk queues, inventory control, dam.

AMS Subject Classification: $60 \mathrm{~K} 10,60 \mathrm{~K} 15,60 \mathrm{~K} 25$.

\section{INTRODUCTION}

In many controlled stochastic models encountered in applications (queues, inventories, dams), a control policy is employed in which a system is restricted in its capability to engage all or a part of its facilities until the total amount of accumulated "work" reaches or exceeds a certain control level (or certain control levels). Some examples include systems with warm-up, orientation, hysteresis service, and multilevel feedback control, bulk queueing systems with service delay discipline, queueing systems with server vacations, inventory control systems, and certain controlled dam mo-

${ }^{1}$ Received: July, 1991. Revised: December, 1991. 
dels.

A bulk queueing system acts similar to a service discipline in which the server can start a new service act only if, after service completion, it finds at least $r(r \geq 1)$ units in the queue; otherwise, the server remains idle until the queue length reaches or exceeds level $r$. It is clear that a preliminary analysis of the first passage problem is necessary, and it is an essential part of any attempt to investigate the functioning of such a system. This fact is illustrated by the authors in [1], where a general control $\mathrm{M}^{X} / \mathrm{G}^{Y} / 1$ bulk queueing system with a service delay discipline of this kind is considered.

In the present article, the authors study a general first passage problem and its applications to the analysis of some stochastic models. Having originated from needs of reliability theory, the first passage problem is traditionally concerned with the distribution of the moment of the first passage (so-called "passage time") of a cumulative random process with single increments over a certain level. In this article, the authors, keeping in mind stochastic model applications, concentrate their attention on the other important aspect of the problem: the distribution of the value of the first excess of a cumulative process (with generally distributed increments) over a fixed level. This random variable is especially important in the analysis of queueing and inventory models with bulk input. In addition, the distribution of the shortage of the first excess, the levels of the first and prefirst excesses, and the moments of the first and pre-first excesses are found. The authors introduce various functionals of the above mentioned processes and manage to express them in terms of a certain function called the "generator." Not only does this generator have a number of fine probabilistic qualities, but it turns out to be a polynomial which considerably facilitates the further analysis (for example, by using factorization methods).

Some results obtained by a direct probability approach can be derived (as it was kindly suggested by Lajos Takács to one of the authors) after some adjustments of more general results from Dynkin [4] and Takács [5]. However, for the sake of simplicity and for an illustration of the methods used in this article, the authors preferred to use in these cases their original proofs. The other results are new. A direct approach for finding a number of various characteristics of the first passage problem, which is developed in the article, is believed to be of independent methodological interest.

The results obtained in this paper are illustrated by a number of examples and, then, are applied to a bulk queueing system with a service delay discipline. 


\section{FORMULATION OF TIE PROBLEM AND GENERAL RESULTS}

All stochastic processes will be considered on a probability space $\{\Omega, \mathcal{F}, P\}$. Let $Z=\sum_{n \geq 0} S_{n} \varepsilon_{\tau_{n}}$ (where $\varepsilon_{a}$ is the Dirac point mass) be a compound random counting measure, such that the counting measures, $\tau=\sum_{n \geq 0} \varepsilon_{\tau_{n}}$ on $\left(\mathbb{R}_{+}, \mathfrak{B}\left(\mathbb{R}_{+}\right)\right)$(where $\mathfrak{B}$ is the Borel $\sigma$-algebra) and $S=\sum_{n=0}^{\infty} \varepsilon_{S_{n}}$ on $(\Psi, \mathfrak{B}(\Psi)), \Psi \subseteq \mathbb{R}_{+}$, be delayed renewal processes, and such that the compound random measure $Z$ be obtained from $\tau$ by position independent marking. We will consider two different cases: $\Psi \subseteq N_{0}$ and $\Psi \sim \mathbb{R}$ (equipped with the usual topology). Observe that, due to its analytic properties and an importance in applications to stochastic models (for example, embedded Markov chains in queueing theory), the discrete case is of main interest, and, consequently, it will be discussed in greater detail than its continuous counterpart.

Therefore, in this section we will study the "critical behavior" of a compound delayed renewal process, $Z$, determined by a delayed renewal process $\tau=\left\{\tau_{n}=t_{0}+t_{1}+\ldots+t_{n} ; n \geq 0\right\}$ on $\mathbb{R}_{+}$, marked by a discrete-valued, delayed renewal process, $S=\left\{S_{n}=X_{0}+X_{1}+\ldots+X_{n} ; n \geq 0\right\}$ on $\Psi$. As mentioned above, we assume that the processes $\tau$ and $S$ are independent. [In the case of their dependence we may arrive at more general results, but we have to indicate what kind of dependence is to be employed. This will not be discussed in this paper.] Let $X_{0}=S_{0}$ be distributed as $P_{X_{0}}=\sum_{i \epsilon \Psi} g_{i} \varepsilon_{i}$ and let $X_{1}$ be distributed as $P_{X_{1}}=\sum_{i \epsilon \Psi} a_{i} \varepsilon_{i}$ (both arbitrary atomic probability measures). The corresponding generating functions are denoted $g(z)=E\left[z^{X_{0}}\right]$ and $a(z)$ $=E\left[z^{X_{1}}\right]$, analytic inside the open unit ball $B(0,1)$ and continuous on its boundary $\partial B(0,1)$, with finite means $\bar{g}=E\left[X_{0}\right]$ and $\bar{a}=E\left[X_{1}\right]$. Without loss of generality we set $t_{0}=0$. We also assume that inter-renewal times $t_{n}=\tau_{n}-\tau_{n-1}$, are described in terms of the common Laplace-Stieltjes transform $V(\theta)=E\left[e^{-\theta t} n\right]$, with the finite mean $\bar{V}=E\left[t_{n}\right], n=1,2, \ldots$.

For a fixed integer $r \geq 1$ we will be interested in the behavior of the process $S$ and some related processes about the level $r$.

The following terminology is introduced and will be used throughout the paper.

\subsection{Definitions.}

(i) Denote $\nu=\inf \left\{k \geq 0: S_{k} \geq r\right\}$ and call it the index of the first excess (above level $r-1$ ).

(ii) Call the random variable $S_{\nu}$ the level of the first excess (above $r-1$ ).

(iii) Call $\eta=S_{\nu}-r$ the magnitude of the first excess of $S$ above level $r-1$.

(iv) Denote $\tilde{\nu}=\sup \left\{k \geq 0: S_{k}<r\right\}=\nu-1$. Call $S_{\tilde{\nu}}$ the pre-first excess level.

( $v$ ) Denote the random variable $\tau_{\nu}$ as the first passage time when $S$ exceeds level $r-1$. 
(vi) Denote $\sigma=r-S_{\widetilde{\nu}}$ and call it the shortage before the first excess of $S$ of level $r$.

We shall be interested primarily in the joint distributions of the first passage time and the random variables listed in $2.1(i-v)$ in terms of the following functionals:

$$
\gamma_{r}(\theta, z)=E\left[e^{-\theta \tau} \nu z^{\nu}\right], \varrho_{r}(\theta, z)=E\left[e^{-\theta \tau} \nu_{z} S_{\nu}\right], g^{(r)}(\theta, z)=E\left[e^{-\theta \tau} \nu_{z} S_{\widetilde{\nu}}\right] .
$$

In addition, introduce the following auxiliary functions

$$
\begin{gathered}
G_{r}(\theta, z)=\sum_{j \geq 0} E\left[e^{-\theta \tau} j_{z}{ }^{S} I_{U_{r-1}}\left(S_{j}\right)\right], \\
G_{r}^{-}(\theta, z)=\sum_{j \geq 1} E\left[e^{-\theta \tau} j_{z}{ }^{S} j_{I_{U^{-1}}}\left(S_{j-1}\right)\right], \\
G_{r}^{+}(\theta, z)=\sum_{j \geq 0} E\left[e^{-\theta \tau} j_{z}{ }^{S} I_{I_{U_{-1}}}\left(S_{j+1}\right)\right],
\end{gathered}
$$

where $U_{p}=\{0,1, \ldots, p\}$, and $I_{A}$ is the indicator function of a set $A$. We call $G_{r}(\theta, z)$ the generator of the first excess level. We shall also use the functionals, which we wish to call the projective functionals, of the following marginal processes:

$$
\gamma_{r}(z)=\gamma_{r}(0, z), g_{r}(z)=\varrho_{r}(0, z), G_{r}(z)=G_{r}(0, z), G_{r}^{-}(z)=G_{r}^{-}(0, z), G_{r}^{+}(z)=G_{r}^{+}(0, z) .
$$

A very important property of $G_{r}(z)$ and $G_{r}^{+}(z)$ is that they are polynomials of $(r-1)$ th degree. As mentioned in the introduction, this fact plays an important role in the analysis of stochastic processes, specifically, it enables one to factor some functionals in polynomials and in known analytic functions.

2.2 Theorem. The joint functional $\gamma_{r}(\theta, z)$ of the first passage time and the index of the first excess can be determined from the following formula:

$$
\gamma_{r}(\theta, z)=1-(1-z V(\theta)) \mathscr{\Phi}_{x}^{r-1}\left\{\frac{g(x)}{(1-x)[1-z V(\theta) a(x)]}\right\}
$$

$$
\mathfrak{I}_{x}^{k}=\lim _{x \rightarrow 0} \frac{1}{k !} \frac{\partial^{k}}{\partial x^{k}}, k \geq 0 .
$$

Proof. Using the routine calculus of indicator functions, we get

$$
\begin{gathered}
\gamma_{r}(\theta, z)=\sum_{j \geq 1}\left\{E\left[e^{-\theta \tau} z^{j} I_{U_{r-1}}\left(S_{j-1}\right) I_{U_{r-1}^{c}}\left(S_{j}\right)\right]+E\left[e^{-\theta \tau} I_{U_{r-1}^{c}}\left(S_{j}\right)\right]\right\} \\
=1+\sum_{j \geq 1} z^{j} E\left[e^{-\theta \tau} I_{U_{r-1}}\left(S_{j-1}\right)\right]-\sum_{j \geq 0} z^{j} E\left[e^{-\theta \tau^{j}} I_{U_{r-1}}\left(S_{j}\right)\right] .
\end{gathered}
$$

Let $\gamma(x, \theta, z)=(1-x) \sum_{p=0}^{\infty} x^{p} \gamma_{p+1}(\theta, z)$. The function $\gamma(x, \theta, z)$ is obviously analytic in the region

$$
\mathrm{C}=B(0,1) \times\{\operatorname{Re}(\theta)>0\} \times B(0,1)
$$

and continuous on $B(0,1) \times\{\operatorname{Re}(\theta) \geq 0\} \times \bar{B}(0,1)$. By the monotone convergence theorem,

$$
\begin{gathered}
\gamma(x, \theta, z)=(1-x) \sum_{j \geq 1} z^{j} E\left[e^{-\theta \tau} j \sum_{p=0}^{\infty} x^{p} I_{U_{p}}\left(S_{j-1}\right)\right] \\
-(1-x) \sum_{j \geq 0} z^{j} E\left[e^{-\theta \tau} j \sum_{p=0}^{\infty} x^{p} I_{U_{p}}\left(S_{j}\right)\right]+1
\end{gathered}
$$




$$
\begin{gathered}
=\sum_{j \geq 1} z^{j} E\left[e^{-\theta \tau} j x^{S} j-1\right]-\sum_{j \geq 0} z^{j} E\left[e^{-\theta \tau} j x^{S_{j}}\right]+1 \\
=\sum_{j \geq 1} z^{j} V^{j}(\theta) g(x) a^{j-1}(x)-\sum_{j \geq 0} z^{j} V^{j}(\theta) g(x) a^{j}(x)+1 \\
=1-(1-z V(z)) \frac{g(x)}{1-z V(\theta) a(x)} .
\end{gathered}
$$

Formula (2.2a) follows after the application of the operator $\mathscr{T}_{x}^{r-1}$ to $\gamma(x, \theta, z)(1-x)^{-1}$.

Formula (2.2a) yields the following pair of corollaries.

2.3 Corollary. The mean value of the index of the first excess can be determined from the formula

$$
\bar{\gamma}_{r}=E[\nu]=\mathscr{D}_{x}^{r-1}\left\{\frac{g(x)}{(1-x)[1-a(x)]}\right\} .
$$

2.4 Corollary. The Laplace-Stieltjes transform of the first passage time $E\left[e^{-\theta \tau_{\nu}}\right]$ equals

$$
E\left[e^{-\theta \tau} \nu\right]=1-(1-V(\theta)) \mathfrak{D}_{x}^{r-1}\left\{\frac{g(x)}{(1-x)[1-V(\theta) a(x)]}\right\} .
$$

Specifically, the mean first passage time is

$$
E\left[\tau_{\nu}\right]=\bar{V} \bar{\gamma}_{r}
$$

2.5 Proposition. The generator $G_{r}(\theta, z)$ of the first excess level can be determined from the following formula:

$$
G_{r}(\theta, z)=\mathscr{T}_{x}^{r-1}\left\{\frac{g(x z)}{(1-x)[1-V(\theta) a(x z)]}\right\}
$$

Proof. Denote

$$
G(x, \theta, z)=(1-x) \sum_{p \geq 0} G_{p+1}(\theta, z) x^{p} .
$$

The function $G(x, \theta, z)$ is obviously analytic in the region $\mathrm{C}$, defined in $(2.2 \mathrm{c})$, and continuous on $B(0,1) \times\{\operatorname{Re}(\theta) \geq 0\} \times \bar{B}(0,1)$. By the monotone convergence theorem,

$$
\begin{gathered}
G(x, \theta, z)=(1-x) \sum_{j \geq 0} E\left[z^{S} j^{-\theta \tau} \sum_{p \geq 0} x^{p} I_{U_{p}}\left(S_{j}\right)\right] \\
=(1-x) \sum_{j \geq 0} E\left[z^{S} e^{-\theta \tau} \sum_{p \geq S_{j}} x^{p}\right]=\sum_{j \geq 0} E\left[e^{-\theta \tau} j_{j}(x z)^{S}\right] \\
=\frac{g(x z)}{1-V(\theta) a(x z)},(x, \theta, z) \in \mathrm{C} .
\end{gathered}
$$

Formula (2.5a) follows from the last equation when we apply the operator $\mathscr{T}_{x}^{r-1}$ to the function $G(x, \theta, z)(1-x)^{-1}$.

The rationale behind the use of the term "generator of the first excess level" comes from the following major theorems and properties, which follow from corollary 2.3 and proposition 2.5.

2.6 Corollary. The "projective" generator $G_{r}(z)$ of the first excess has the following property: 


$$
G_{r}(1)=\bar{\gamma}_{r}=E[\nu]
$$

2.7 Proposition. $G_{r}^{-}(\theta, z)$ can be obtained from the following formula:

$$
G_{r}^{-}(\theta, z)=\mathscr{T}_{x}^{r-1}\left\{\frac{V(\theta) a(z) g(x z)}{(1-x)[1-V(\theta) a(x z)]}\right\}=a(z) V(\theta) G_{r}(z) .
$$

Proof. Let

$$
G^{-}(x, \theta, z)=(1-x) \sum_{p \geq 0} G_{p+1}^{-}(\theta, z) x^{p} .
$$

Then, by reasonings similar to those in the proof of theorem 2.2 , we get

$$
G^{-}(x, \theta, z)=\frac{V(\theta) a(z) g(x z)}{1-V(\theta) a(x z)},(x, \theta, z) \in \mathrm{e} .
$$

The statement of the proposition follows.

2.8 Proposition. The functional $G_{r}^{+}(\theta, z)$ can be obtained from the following formula:

$$
G_{r}^{+}(\theta, z)=\mathscr{D}_{x}^{r-1}\left\{\frac{a(x) g(x z)}{(1-x)[1-V(\theta) a(x z)]}\right\} \text {. }
$$

Proof. The proof of proposition 2.8 is an analog to that of proposition 2.7.

2.9 Theorem. The functional $\mathrm{g}_{r}(\theta, z)$ of the first passage time and the first excess level can be expressed in terms of the generator of the first excess as

$$
g_{r}(\theta, z)=g(z)-[1-V(\theta) a(z)] G_{r}(\theta, z) .
$$

Proof. From

we have that

$$
E\left[e^{-\theta \tau} \nu z^{S} \nu\right]=E\left[z^{S} I_{U_{r-1}^{c}}\left(S_{0}\right)\right]+E\left[e^{-\theta \tau} \nu z^{S} \nu I_{U_{r-1}}\left(S_{0}\right)\right]
$$

$$
E\left[e^{-\theta \tau} z_{z}^{S}\right]=\sum_{i=r}^{\infty} g_{i} z^{i}+\sum_{j \geq 1} E\left[e^{-\theta \tau_{j} S_{j}} I_{U_{r-1}}\left(S_{j-1}\right) I_{U_{r-1}^{c}}\left(S_{j}\right)\right] .
$$

Using the routine calculus for indicator functions we get

$$
\begin{gathered}
E\left[z^{S} \nu\right]=\sum_{i=r}^{\infty} g_{i} z^{i}+\sum_{j \geq 1} E\left[e^{-\theta \tau_{j}} z_{z}^{S} j_{U_{r-1}}\left(S_{j-1}\right)\right] \\
-\sum_{j \geq 1} E\left[e^{-\theta \tau} j_{z}{ }^{S} j_{U_{r-1}}\left(S_{j}\right)\right] .
\end{gathered}
$$

The third sum in $(2.9 \mathrm{a})$ is obviously $G_{r}(\theta, z)$ less $E\left[z^{S_{0}} I_{U_{r-1}}\left(S_{0}\right)\right]=\sum_{i=0}^{r-1} g_{i} z^{i}$, and the second sum in $(2.9 \mathrm{a})$ is $G_{r}^{-}(\theta, z)$. The statement of the theorem follows from propositions 2.5 and 2.7 .

2.10 Corollary. The mean value of the first excess level can be determined from the following formula:

$$
\bar{g}_{r}=E\left[S_{\nu}\right]=\bar{g}+\bar{a} \bar{\gamma}_{r} .
$$

Proof. The validity of formula $(2.10 \mathrm{a})$ is due to corollary 2.6 , theorem 2.9 and routine calculus. 
2.11 Remark. Observe that the "total magnitude" $S_{\nu}-S_{0}$ of the first excess level has the mean value $\bar{\varrho}_{r}-\bar{g}$ and, due to $(2.10 \mathrm{a})$, equals $\bar{a} \bar{\gamma}_{r}$, i.e., the product of the mean "batch" size and the mean value of the index. Thus, it seems as if we could proceed with Wald's formula to get the same result. However, it would be unjustified, since, in $S_{\nu}, X_{1}, \ldots, X_{\nu}$ are not independent of $\nu$ (as trivial counterexamples show); consequently, a similar factorization of other functionals of $S_{\nu}-S_{0}$ is not possible. This tells us that Wald's equation apparently holds true for weaker sufficient conditions.

2.12 Theorem. The functional $\mathrm{g}^{(r)}(\theta, z)$ of the pre-first passage time and the pre-first excess level can be determined from the following formula:

$$
\begin{aligned}
& g^{(r)}(\theta, z)=G_{r}(\theta, z)-G_{r}^{+}(\theta, z)+P\left\{S_{0} \geq r\right\} \\
= & \mathscr{T}_{x}^{r-1}\left\{\frac{[1-a(x)] g(x z)}{(1-x)[1-V(\theta) a(x z)]}\right\}+P\left\{S_{0} \geq r\right\},
\end{aligned}
$$

where we define $S_{\widetilde{\nu}}=0$ and $\tau_{\widetilde{\nu}}=0$ on the set $\left\{S_{0} \geq r\right\}$.

Proof. The functional $\mathrm{g}^{(r)}(\theta, z)$ can be decomposed as

$$
E\left[e^{-\theta \tau} \widetilde{\nu} z^{S} \widetilde{\nu}\right]=E\left[e^{-\theta \tau} \widetilde{\nu} z^{S} \widetilde{\nu} I_{U_{r-1}}\left(S_{0}\right)\right]+E\left[e^{-\theta \tau_{\widetilde{\nu}} z^{S} \widetilde{\nu}} I_{U_{r-1}^{c}}\left(S_{0}\right)\right]
$$

Then, formula $(2.12 b)$ is reduced to

$$
E\left[e^{-\theta \tau} \widetilde{\nu}{ }_{z}^{S} \widetilde{\nu}\right]=E\left[e^{-\theta \tau} \widetilde{\nu} z_{\widetilde{\nu}}^{S} I_{U_{r-1}}\left(S_{0}\right)\right]+P\left\{S_{0} \geq r\right\}
$$

The expected value on the right-hand side of $(2.12 \mathrm{c})$ can be modified as follows.

$$
\begin{aligned}
& E\left[e^{-\theta \tau} \widetilde{\nu} z^{S} \widetilde{\nu} I_{U_{r-1}}\left(S_{0}\right)\right]=\sum_{j \geq 0} E\left[e^{-\theta \tau_{j}} z^{S}{ }^{j} I_{U_{r-1}}\left(S_{j}\right) I_{U_{r-1}^{c}}\left(S_{j+1}\right)\right] \\
& =\sum_{j \geq 0} E\left[e^{-\theta \tau} j_{z}{ }^{S} I_{U_{r-1}}\left(S_{j}\right)\right]-\sum_{j \geq 0} E\left[e^{-\theta \tau_{j}} z^{S}{ }_{j} I_{U_{r-1}}\left(S_{j+1}\right)\right] \text {. }
\end{aligned}
$$

Thus,

$$
E\left[e^{-\theta \tau} \tilde{\nu}_{z}^{S} \widetilde{\nu}_{U_{r-1}}\left(S_{0}\right)\right]=G_{r}(\theta, z)-G_{r}^{+}(\theta, z)
$$

Finally, formula (2.12a) follows from propositions 2.5 and 2.8 .

\section{CONTINUOUS-VALUED PROCESSES}

In this section we obtain joint functionals of the first passage time and the first excess level above some positive real number $s$. We assume that $Z=\sum_{n \geq 0} S_{n} \varepsilon_{\tau_{n}}$ is a compound random measure obtained from $\tau$ by position independent marking, where $S=\sum_{n \geq 0} \varepsilon_{S_{n}}$ is a counting measure on $\left(\mathbb{R}_{+}, \mathscr{B}_{(}\left(\mathbb{R}_{+}\right)\right)$such that $S$ is a delayed renewal process. We denote

$$
\begin{gathered}
V_{0}(\theta)=E\left[e^{-\theta \tau} 0\right], V(\theta)=E\left[e^{-\theta t} 1\right], \gamma(\vartheta)=E\left[e^{-\vartheta S_{0}}\right], \alpha(\vartheta)=E\left[e^{-\vartheta X_{1}}\right], \\
\mathcal{G}_{s}(\theta, \vartheta)=E\left[e^{-\theta \tau} \nu-\vartheta S_{\nu}\right] .
\end{gathered}
$$

and 
We evaluate $g_{s}(\theta, \vartheta)$ in terms of the Laplace transform by using methods similar to those in the previous section. And we let

$$
g(\theta, \vartheta, t)=\int_{s=0}^{\infty} e^{-t s} g_{s}(\theta, \vartheta) d s, R e(t)>0 .
$$

3.1 Theorem. The functional $\mathrm{g}(\theta, \vartheta, t)$ can be determined from the following expression:

$$
\mathcal{g}(\theta, \vartheta, t)=\frac{1}{t} V_{0}(\theta)\left\{\gamma(\vartheta)-\gamma(t+\vartheta) \frac{1-V(\theta) \alpha(\vartheta)}{1-V(\theta) \alpha(t+\vartheta)}\right\}
$$

Proof. By the monotone convergence theorem,

$$
\begin{gathered}
\mathcal{g}(\theta, \vartheta, t)=\sum_{j \geq 1} E\left[e^{-\theta \tau} j \int_{s=0}^{\infty} e^{-t s} e^{-\vartheta S} j_{[0, s)}\left(S_{j-1}\right) I_{[s, \infty)}\left(S_{j}\right) d s\right] \\
+E\left[e^{-\theta \tau \nu-\vartheta S} \nu \int_{s=0}^{\infty} e^{-t s} I_{[s, \infty)}\left(S_{0}\right) d s\right]
\end{gathered}
$$

This reduces to

$$
\begin{gathered}
\mathcal{G}(\theta, \vartheta, t)=\sum_{j \geq 1} E\left[e^{-\theta \tau_{j}} e^{-\vartheta S_{j}} \int_{s=S_{j-1}}^{S_{j}} e^{-t s} d s\right]+E\left[e^{-\theta \tau_{0}} e^{-\vartheta S_{0}} \int_{s=0}^{S_{0}} e^{-t s} d s\right] \\
=\frac{1}{t} \sum_{j \geq 1} V_{0}(\theta) V^{j}(\theta) \gamma(t+\vartheta) \alpha^{j-1}(t+\vartheta)[\alpha(\vartheta)-\alpha(t+\vartheta)]+\frac{1}{t} V_{0}(\theta)[\gamma(\vartheta)-\gamma(t+\vartheta)] \\
=\frac{1}{t} V_{0}(\theta)\left\{\gamma(\vartheta)-\gamma(t+\vartheta) \frac{1-V(\theta) \alpha(\vartheta)}{1-V(\theta) \alpha(t+\vartheta)}\right\} .
\end{gathered}
$$

\subsection{Examples.}

(i) For $\theta=0$ and for $\gamma(\vartheta)=1$, we get from (3.1a) the functional of the first excess level as

$$
\mathfrak{g}(0, \vartheta, t)=\frac{1}{t} \frac{\alpha(\vartheta)-\alpha(t+\vartheta)}{1-\alpha(t+\vartheta)}
$$

Consider (3.2a) under the additional assumption that

$$
\alpha(\vartheta)=\frac{\alpha}{\alpha+\vartheta}
$$

(as the Laplace-Stieltjes transform of an exponential distribution with mean $1 / \alpha$ ), which reduces $(3.2 \mathrm{a})$ to

Then,

$$
\mathcal{g}(0, \vartheta, t)=\frac{\alpha}{\vartheta(\alpha+\vartheta)} \cdot \frac{\vartheta}{t+\vartheta}
$$

$$
\oint_{s}(0, \vartheta)=E^{0}\left[e^{-\vartheta S_{\nu}}\right]=e^{-\vartheta s} \frac{\alpha}{\alpha+\vartheta}
$$

and, therefore, $S_{\nu}$ has the probability density function $\alpha e^{-\alpha(x-s)} I_{[s, \infty)}(x)$.

(ii) For $\vartheta=0$ and $V_{0}(\theta)=1$, we obtain the formula for the functional of the first passage time,

$$
\mathcal{G}(\theta, 0, t)=\frac{V(\theta)[1-\alpha(t)]}{t[1-\alpha(t) V(\theta)]}
$$


which under assumption $(3.2 \mathrm{~b})$ reduces to

$$
\mathcal{g}(\theta, 0, t)=\frac{V(\theta)}{\alpha[1-V(\theta)]+t} .
$$

Then, the inverse of the Laplace transform in $t$ gives the Laplace-Stieltjes transform of the first passage time of level $s$ :

$$
\oint_{s}(\theta, 0)=V(\theta) \exp \{-\alpha s[1-V(\theta)]\}
$$

\section{SPECIAL CASES AND APPLICATIONS}

4.1 Remark. The special case of formula (2.2a) for $\theta=0$ can be derived by using different arguments. Denoting $\left\{S_{-1} \geq r\right\}=\emptyset$, we obviously have, for all $n=0,1, \ldots$, that

$$
\{\nu=n\}=\left\{S_{n} \geq r\right\} \backslash\left\{S_{n-1} \geq r\right\} .
$$

Consequently

$$
P\{\nu=n\}=P\left\{S_{n} \geq r\right\}-P\left\{S_{n-1} \geq r\right\}
$$

Thus,

$$
\begin{gathered}
\gamma_{r}(z)=(1-z) h_{r}(z), \\
h_{r}(z)=\sum_{n=0}^{\infty} z^{n} P\left\{S_{n} \geq r\right\} .
\end{gathered}
$$

Let $h(x, z)=\sum_{k=0}^{\infty} h_{k}(z) x^{k}$. Then, after some transformations we obtain that

$$
h(x, z)=\frac{1}{(1-z)(1-x)}-\frac{x g(x)}{(1-x)[1-z a(x)]} .
$$

The formula,

$$
\gamma_{r}(z)=1-(1-z) \mathfrak{D}_{x}^{r-1}\left\{\frac{g(x)}{(1-x)[1-z a(x)]}\right\}
$$

follows from (4.1a) and (4.1b) along with the use of the operator (2.2b).

In what follows we assume that $g(z)=z^{i}$ (i.e. $S_{0}=i$ a.s.). We then label the corresponding functionals of all discussed random variables with index " $i$."

4.2 Corollary. The joint functional $\gamma_{r}^{(i)}(\theta, z)$ of the first passage time and the index of the first excess level satisfies the following formula:

$$
\gamma_{r}^{(i)}(\theta, z)=\left\{\begin{array}{cl}
V(\theta) z \mathscr{I}_{x}^{r-i-1}\left\{\frac{1-a(x)}{(1-x)(1-z V(\theta) a(x))}\right\}, & i<r \\
1, & i \geq r .
\end{array}\right.
$$

Proof. From (2.2a) it directly follows that 


$$
\begin{gathered}
\gamma_{r}^{(i)}(\theta, z)=1-(1-V(\theta) z) \Phi_{x}^{r-i-1}\left\{\frac{1}{(1-x)[1-z V(\theta) a(x)]}\right\}, i<r, \\
\gamma_{r}^{(i)}(\theta, z)=1, i \geq r
\end{gathered}
$$

after noticing that

$$
\mathscr{D}_{x}^{r-1}\left\{x^{i} f(x)\right\}=\left\{\begin{array}{cc}
\mathscr{T}_{x}^{r-i-1}\{f(x)\}, & i<r \\
0, & i \geq r .
\end{array}\right.
$$

It is readily seen that formulas $(4.2 \mathrm{a})$ and $(4.2 \mathrm{~b}-4.2 \mathrm{c})$ are equivalent.

From formulas (4.2b-4.2c), we immediately obtain the mean value of the first excess index:

$$
E[\nu]=\bar{\gamma}_{r}^{(i)}=\left\{\begin{array}{cc}
\mathscr{D}_{x}^{r-i-1}\left\{\frac{1}{(1-x)[1-a(x)]}\right\}, & i<r \\
0, & i \geq r .
\end{array}\right.
$$

4.4 Corollary. The generating function $\mathcal{G}_{r}^{(i)}(z)$ of the first excess level is determined by the following fornula:

$$
\mathscr{G}_{r}^{(i)}(z)=\left\{\begin{array}{cc}
z^{i} \Im_{x}^{r-i-1}\left\{\frac{a(z)-a(x z)}{(1-x)(1-a(x z))}\right\}, & i<r \\
z^{i}, & i \geq r .
\end{array}\right.
$$

By using change of variables in (4.4a) we can transform it into an equivalent expression

$$
g_{r}^{(i)}(z)=\left\{\begin{array}{cc}
z^{r} \mathfrak{T}_{x}^{r-i-1}\left\{\frac{a(z)-a(x)}{(z-x)[1-a(x)]}\right\}, & i<r \\
z^{i}, & i \geq r .
\end{array}\right.
$$

Proof of corollary 4.4. Formula (4.4a) follows from (2.3a) by direct computations. Alternatively, formula $(4.4 \mathrm{a})$ or its equivalent $(4.4 \mathrm{~b})$ can be derived from entirely different probability arguments that can be of independent interest.

Our preliminary target is the generating function $g_{r}^{(i)}(z)$ of the magnitude $\eta=\eta_{r}^{(i)}$ of the first excess level with the above assumption that $S_{0}=i$ a.s. Since obviously $\eta_{r}^{(i)}=\eta_{r}^{(0)}{ }_{i}$, we can operate with $\eta_{r}^{(0)}$ to determine $g_{r}^{(0)}(z)$. Then we shall return to the general case by restoring corresponding indices. Introduce the following notation:

$$
\begin{gathered}
q_{n s}=P\left\{S_{n}=s\right\}, q_{n 0}=0, l_{s}=\sum_{n=0}^{\infty} q_{n s}, l_{0}=1, \\
b_{n}(z)=\sum_{m=1}^{\infty} a_{n+m} z^{m-1}, k, n, s=0,1, \ldots
\end{gathered}
$$

From direct probability arguments it follows that $P\left\{\eta_{r}^{(0)}=k\right\}=\sum_{s=0}^{r-1} l_{s} a_{r+k-s}$, and thus

$$
g_{r}^{(0)}(z)=\sum_{s=0}^{r-1} b_{r-s-1}(z) l_{s} .
$$

On the other hand, the series $\sum_{n=0}^{\infty} b_{n}(z) x^{n}$ (with $b_{n}(z)$ defined in (4.4d)) converges in the region 
$C=\{\|x\|<\|z\| \leq 1\} ;$ and in $C$ we have that

$$
\sum_{n=0}^{\infty} b_{n}(z) x^{n}=\frac{a(z)-a(x)}{z-x} \text { and } \sum_{s=0}^{\infty} l_{s} x^{s}=\frac{1}{1-a(x)} .
$$

Applying (4.4e) and (4.4f) to $g(x, z)=\sum_{s=1}^{\infty} g_{s}^{(0)}(z) x^{r}$, we obtain that in region $C$

$$
\mathrm{g}(x, z)=\frac{x[a(z)-a(x)]}{(z-x)[1-a(x)]} .
$$

Therefore, $g_{r}^{(0)}(z)=\mathscr{D}_{x}^{r-1}\left\{\frac{a(z)-a(x)}{(z-x)[1-a(x)]}\right\}$ which implies that

$$
g_{r}^{(i)}(z)=\mathscr{T}_{x}^{r-i-1}\left\{\frac{a(z)-a(x)}{(z-x)[1-a(x)]}\right\},
$$

which by definition of $S_{\nu}$ yields (4.4a).

[Observe that since both the left- and right-hand sides of $(4.4 \mathrm{~g})$ are analytic functions in the region $B(0,1) \times B(0,1)$ and since they coincide in the region $C$, by the uniqueness theorem for analytic functions, these two functions also coincide in the whole region $B(0,1) \times B(0,1)$.]

Although the following result could follow from (2.12a), we shall use another direct method.

4.5 Corollary. The generating function $E\left[z^{\sigma}\right]=C_{r}^{(i)}(z)$ of the shortage before the first excess is determined from the following formula:

$$
C_{r}^{(i)}(z)=z_{x}^{r-i-1}\left\{\frac{1-a(x z)}{(1-x z)[1-a(x z)]}\right\}, i=0, \ldots, r-1 .
$$

Proof. For convenience denote $\sigma_{r}^{(i)}=\sigma=r-S_{\widetilde{\nu}}$. Because $\sigma_{r}^{(i)}=\sigma_{0}^{(r-i)}$ we can operate with $C_{r}^{(0)}(z)$ and then restore the original indices.

By direct probability arguments and, using notation (4.4c), we have

$$
C_{r}^{(0, k)}=P\left\{\sigma_{r}^{(0)}=k\right\}=\sum_{j \geq 0} \sum_{n \geq 0} q_{j, r-k} a_{k+n}=l_{r-k} f_{k},
$$

where $f_{k}=\sum_{n \geq 0} a_{k+n}$. Thus,

$$
C_{0}(x, z)=\sum_{r \geq 1} C_{r}^{(0)}(z) x^{r}=\sum_{n \geq 1} f_{n}(x z)^{n} \sum_{m \geq 1} l_{m} x^{m} .
$$

Now, since obviously

$$
\sum_{m \geq 1} f_{m} u^{m}=u \frac{1-a(u)}{1-u}
$$

and by (4.4f), we have that

Therefore,

$$
C_{0}(x, z)=\frac{x z[1-a(x z)]}{(1-x z)[1-a(x)]}
$$

and formula (4.5a) follows.

$$
C_{r}^{(0)}(z)=z \mathscr{I}_{x}^{r-1}\left\{\frac{1-a(x z)}{(1-x z)[1-a(x)]}\right\}
$$

4.6 Corollary. The generator of the first excess level can be determined from the following formula: 


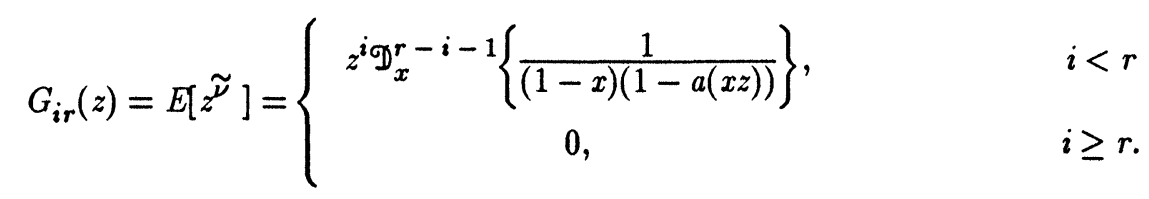

4.7 Corollary. The generating function $\mathrm{g}_{r}^{(i)}(z)$ of the level of the first excess can be expressed in terms of the generator $G_{i r}(z)$ of the first excess level by the following relation:

$$
g_{r}^{(i)}(z)=z^{i}-(1-a(z)) G_{i r}(z), i=0,1, \ldots \text {. }
$$

\subsection{Examples.}

(i) Consider a trivial case when $a_{i}(z)=a(z)=z$. From $(4.4 \mathrm{~g})$ we have that $\mathrm{g}(x, z)=\frac{x}{1-x}$ which yields that $g_{r}^{(0)}(z)=1$, and thus $g_{r}^{(i)}(z)=z^{r}$, as it should be.

(ii) Let $a_{i}(z)=a(z)=z^{2}$. Then, from (4.4g) we have $g(x, z)=\frac{x(z+x)}{1-x^{2}}$ which yields that $g_{r}^{(0)}(z)=z^{r(\bmod 2)}$, and thus $\mathrm{G}_{r}^{(i)}(z)=z^{r+[(r-i)(\bmod 2)]}$ as it should be.

(iii) Let $a_{i}(z)=a(z)=p z+q z^{2} \quad(p>0, p+q=1)$. Then, from (4.4g) it follows that $\mathrm{g}(x, z)=\frac{x[p+q(z+x)]}{(1-x)[1+x q]}$, which after elementary transformations yields

$$
\mathrm{g}_{r}^{(i)}(z)=z^{r}+z^{r} \frac{1-z}{1-z_{1}}\left[z_{1}^{i-r}-1\right], \text { where } z_{1}=-\frac{1}{q} .
$$

(iv) Suppose that batches $X_{1}, X_{2}, \ldots$, are distributed geometrically. In other words, let $a_{i}(z)=a(z)=p z(1-q z)^{-1}$. Then, from $(4.4 \mathrm{~g})$ we have $g(x, z)=\frac{p x}{(1-q z)(1-x)}$ and thus

$$
g_{r}^{(0)}(z)=p(1-q z)^{-1},
$$

which yields $g_{r}^{(i)}(z)=z^{r} p(1-q z)^{-1}$.

4.9 Remark. Observe that the random variable $\eta_{r}^{(i)}=S_{\nu}-r$ is memoryless in this special case. This can be rigorously formulated as follows.

Let $S=\sum_{n \geq 0} \varepsilon_{S_{n}}$ be a delayed renewal process on $\Psi \subseteq \mathbb{N}_{0}$, such that $S_{0}=i$ a.s. and the magnitudes $S_{1}-S_{0}, S_{2}-S_{1}, \ldots$ are geometrically distributed with the common generating function $p z(1-q z)^{-1}$. Then the distribution of the magnitude $\eta_{r}^{(i)}$, of the first excess of level, is independent of $i$ and $r$, and it is also distributed geometrically with the generating function given by formula (4.8a).

4.10 Example. We shall apply the results of section 2 to a special case of one stochastic queueing model (studied in Abolnikov and Dshalalow [1]). Consider a single-server queueing system with an infinite waiting room and a compound Poisson input (described by the compound counting measure $Z=\sum_{n=0}^{\infty} S_{n} \varepsilon_{\tau_{n}}$ ) and a queue length dependent service delay discipline. According to this discipline, the server immediately starts the next service act if the queue length is at least $r$; in 
this case all available units, or $R$ (capacity of the server) of them, whichever is less, are taken for service. Otherwise, the server delays the service act until the number of units in the queue reaches or exceeds level $r$.

Let $\left\{\Omega, \mathcal{F},\left(P^{x}\right)_{x \in \Psi}, Q(t) ; t \geq 0\right\} \rightarrow \Psi=\{0,1, \ldots\}$ be a stochastic process describing the number of units at time $t$ in this queueing system; and let $\sum_{n \geq 0} \varepsilon_{T_{n}}\left(T_{0}=0\right)$ be a counting measure on $\left(\mathbb{R}_{+}, \mathscr{B}\left(\mathbb{R}_{+}\right)\right)$which gives the sequence of successive completions of service; and let $Q_{n}=Q\left(T_{n}+0\right)$. If at time $T_{n}+0$ the queue length, $Q_{n}$, is at least $r$ (a positive integer less than or equal to $R$ ), the server takes a batch of units of size $R$ (a positive integer denoting the capacity of the server) from the queue and then serves it during a random length of time $\sigma_{n+1}$. Otherwise, the server idles until the queue length for the first time reaches or exceeds the level $r$.

Let $X_{1}, X_{2}, \ldots$ be the sizes of successive groups of units arriving at the system after $T_{0}$ and let $S_{k}=X_{0}+X_{1}+\ldots+X_{k}$, where $X_{0}=Q_{0}$. Then, given $Q_{0}, S=\left\{S_{k} ; k \in \mathbb{N}_{0}\right\}$ is an integer-valued delayed renewal process. Recall that $\nu=\nu_{0}=\inf \left\{k \geq 0: S_{k} \geq r\right\}$ denotes the random index when the process $S$ first reaches or exceeds level $r$ and the queue length is $Q_{0}$. Thus, $\tau_{\nu_{0}}$ is the first passage time of the queueing process over level $r$ by the queueing process after time $T_{0}$.

Define

$$
\theta_{n}\left(Q_{n}\right)=\theta_{n}=\left\{\begin{array}{cc}
\tau_{\nu_{n}}, & X_{0}=Q_{n}<r \\
T_{n}, & X_{0}=Q_{n}=S_{n} \geq r .
\end{array}\right.
$$

Then, at the instant $\theta_{n}$ of the first passage time, the server is supposed to take a batch of the size $\min \left\{Q\left(\theta_{n}\right), R\right\}$ for service. In other words, if $Q_{n} \geq r, T_{n+1}-T_{n}$ coincides with length of service time $\sigma_{n+1}$ of the $n+1$ st batch. If $Q_{n}<r$ the interval $\left(T_{n}, T_{n+1}\right]$ contains the waiting time for $X_{1}+\ldots+X_{\nu_{n}}$ units to arrive and the actual service time $\sigma_{n+1}$. In both cases we assume that $\sigma_{n+1}$ has a probability distribution function $B$ with a finite mean $b$.

Finally, we can abbreviate the definition of the servicing process tr rough the following relation for $\left\{Q_{n}\right\}$ :

$$
Q_{n+1}= \begin{cases}\left(S_{\nu_{n}}-R\right)^{+}+Z\left(\sigma_{n+1}\right), & Q_{n}<r \\ \left(Q_{n}-R\right)^{+}+Z\left(\sigma_{n+1}\right), & Q_{n} \geq r .\end{cases}
$$

From relation (4.10a) it follows that the process $\left\{\Omega, \mathcal{F},\left(P^{x}\right)_{x \in \Psi}, Q(t) ; t \geq 0\right\} \rightarrow \Psi$ has at $T_{n}, n \geq 1$, the locally strong Markov property (see Dshalalow [3]). Thus $\left\{\Omega, \mathcal{F},\left(P^{x}\right)_{x \in \Psi}, Q_{n} ; n \in \mathbb{N}_{0}\right\} \rightarrow \Psi$ is a homogeneous Markov chain with transition probability matrix denoted by $A=\left(a_{i j}\right)$.

In [1] it was shown that the generating function $A_{i}(z)$ of the $i$ th row of the matrix $A$ can be determined from the following formulas: 


$$
\begin{gathered}
A_{i}(z)=K(z) H_{i}^{(r, R)}(z), \quad \text { where } \\
H_{i}^{(r, R)}(z)=z^{-R} \mathcal{G}_{r}^{(i)}(z)+\mathscr{D}_{y}^{R-1}\left\{\frac{\mathcal{G}_{r}^{(i)}(y)-z^{-R} \mathcal{G}_{r}^{(i)}(y z)}{1-y}\right\}, i \in \Psi
\end{gathered}
$$

$K(z)=\beta(\lambda-\lambda a(z))$, where $\beta(\theta), R e(\theta) \geq 0$, forms the Laplace-Stieltjes transform of the probability distribution function $B$, and $G_{r}^{(i)}$ satisfies formula (4.4a) or (4.4b).

Observe that since $g_{r}^{(i)}(z)=z^{i}$, for $i \geq r$, formula $(4.9 \mathrm{~b})$ reduces to

$$
H_{i}^{(r, R)}(z)=z^{(i-R)^{+}}, i \geq r \text {. }
$$

It can be shown that $A$ is reduced to a form of the homogeneous $\Delta_{R}$-matrix, which is a special case of a $\Delta_{m, n}$-matrix introduced and studied in [2]. There the stochastic matrix $A=\left(a_{i j}\right.$; $i, j \in \Psi=\{0,1, \ldots\})$ is called a homogeneous $\Delta_{R}$-matrix if it is of the form $A=\left(a_{i j}: i, j \in \Psi\right.$ : $\left.a_{i j}=k_{j-i+r}, i>R, j \geq i-R ; a_{i j}=0, i>R, j<i-R\right)$, where $\sum_{j=0}^{\infty} k_{i} \varepsilon_{i}$ is an atomic probability measure.

In [2] it was shown that the embedded queueing process $Q_{n}$ is ergodic if and only if $\alpha \lambda b<R$. Under this condition, the generating function $P(z)$ of the invariant probability measure $\boldsymbol{P}$ of the operator $A$ is determined by the following formula:

$$
P(z)=\frac{K(z) \sum_{i=0}^{R} p_{i}\left\{z^{R} H_{i}^{(r, R)}(z)-z^{i}\right\}}{z^{R}-K(z)},
$$

where $H_{i}^{(r, R)}$ satisfies $(4.9 \mathrm{~b})$. In addition, it was shown in [1] that the probabilities $p_{0}, \ldots, p_{R}$ form the unique solution of the following system of $R$ linear equations:

$$
\left.\sum_{i=0}^{R} p_{i} \frac{d^{k}}{d z^{k}}\left\{K(z) H_{i}^{(r, R)}(z)-z^{i}\right\}\right|_{z=z_{s}}=0, k=0, \ldots, k_{s}-1, s=1, \ldots, S .
$$

In the above, the values of $z_{s}$, for $s=1, \ldots, S$, are $R$ roots of the function $z \mapsto z^{R}-K(z)$ in the region $\bar{B}(0,1) \backslash\{1\}$ with their multiplicities $k_{s}$ such that $\sum_{s=1}^{S-1} k_{s}=R-1$. Also, the $(R+1)$ th equation is as follows

$$
\sum_{i=0}^{R} p_{i}\left(\bar{\gamma}_{r}^{(i)}-i+\mathscr{T}_{y}^{R-1}\left\{\frac{g_{r}^{(i)}(y)}{(1-y)^{2}}\right\}\right)=R-\alpha \lambda b .
$$

Acknowledgement. The authors are very grateful to Professors Lajos Takács and Boris Pittel for their kind advises and helpful comments. Professor Don Konwinski provided a very careful proofreading of the paper and gave a number of useful suggestions. The authors thank also him. 


\section{REFERENCES}

[1] Abolnikov, L. and Dshalalow, J., On a multilevel controlled bulk queueing system with $(r, R)$-service delay discipline, submitted to Annals of Appl. Prob., 1991.

[2]. Abolnikov, L. and Dukhovny A., Markov chains with transition delta-matrix: ergodicity conditions, invariant probability measures and applications, Journ. Appl. Math. Stoch. Analysis, 4, No. 4, 335-355, 1991.

[3]. Dshalalow, J., On modulated random measures. Journ. Appl. Math. Stoch. Analysis, 4, No.4, 305-312, 1991.

[4]. Dynkin, E., Some limit theorems for sums of independent random variables with infinite mathematical expectations, Izv. Akad. Nauk SSSR, Ser. Math. 19, 247-266, 1955 [or in Selected Translations in Mathematical Statistics and Probability, IMS and AMS, 1, 171-189, 1961].

[5]. Takács, L., On fluctuations of sums of random variables, Advances in Mathematics, 2, 45-93, 1978. 


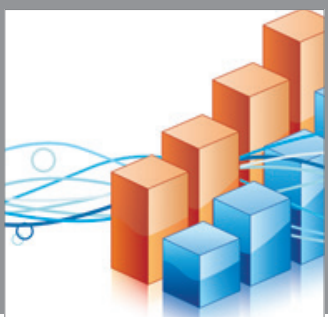

Advances in

Operations Research

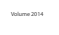

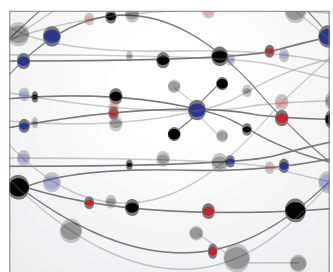

\section{The Scientific} World Journal
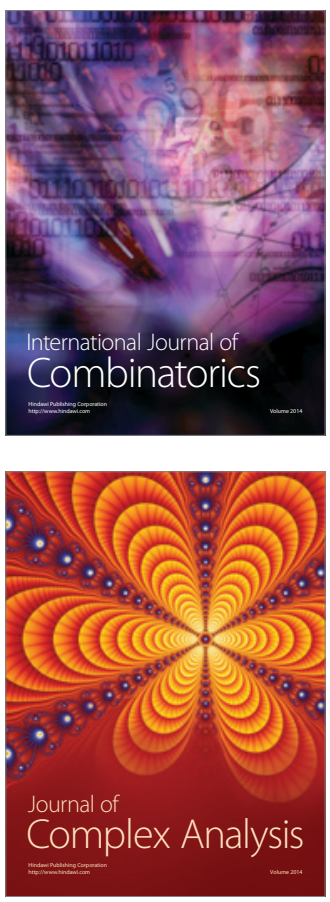

International Journal of

Mathematics and

Mathematical

Sciences
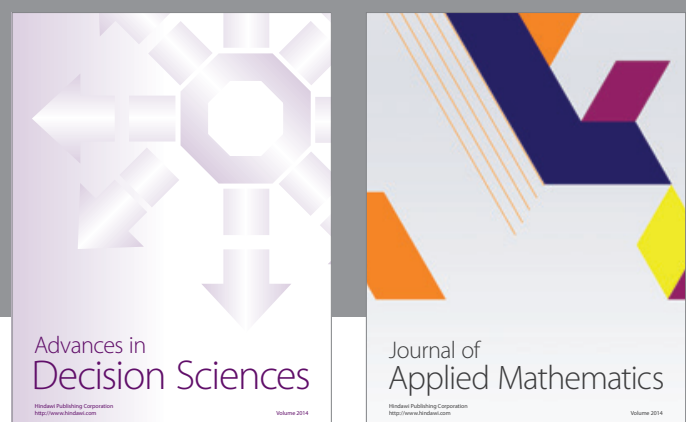

Journal of

Applied Mathematics
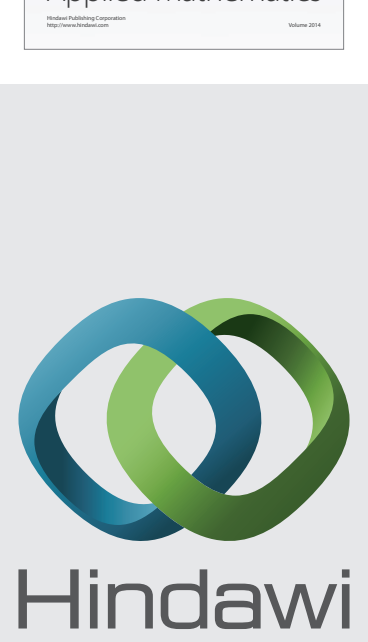

Submit your manuscripts at http://www.hindawi.com
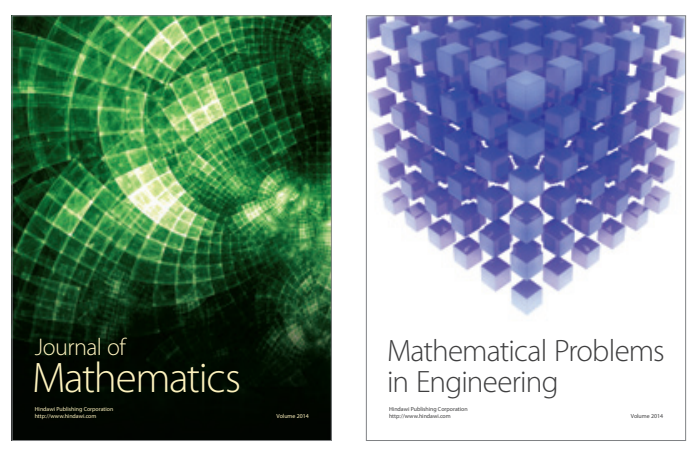

Mathematical Problems in Engineering
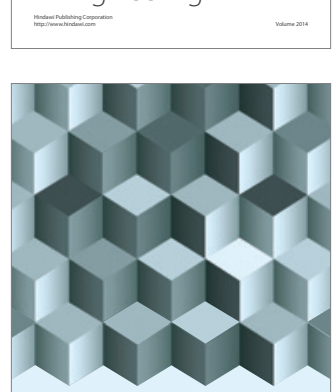

Journal of

Function Spaces
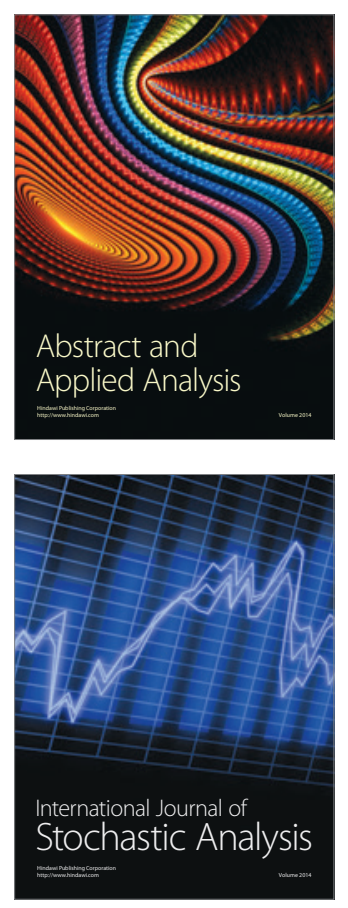

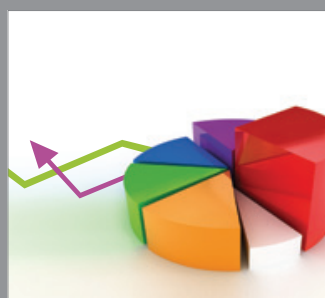

ournal of

Probability and Statistics

Promensencen
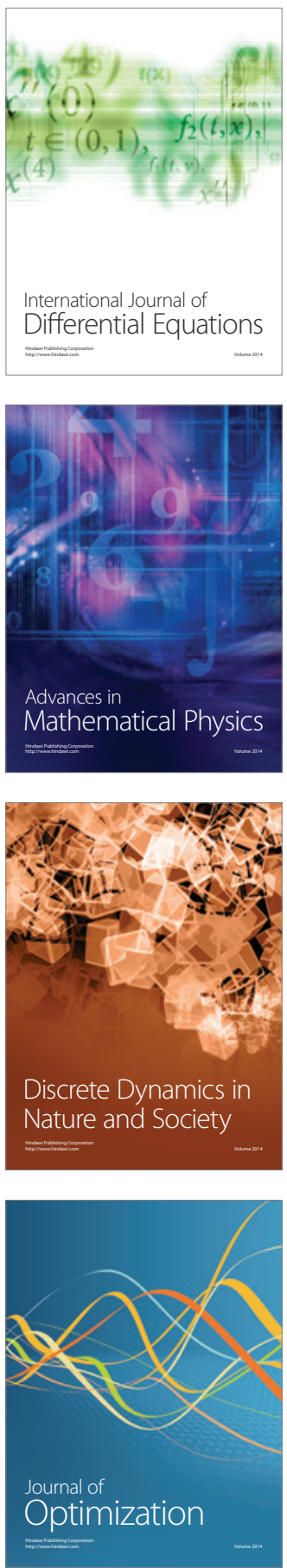